\title{
Bile acid aspiration and the development of bronchiolitis obliterans after lung transplantation
}

\author{
Frank D'Ovidio, MD, Marco Mura, MD, Melanie Tsang, MSc, Thomas K. Waddell, MD, Michael A. Hutcheon, MD, \\ Lianne G. Singer, MD, Denis Hadjiliadis, MD, Cecilia Chaparro, MD, Carlos Gutierrez, MD, Andrew Pierre, MD, \\ Gail Darling, MD, Mingyao Liu, PhD, and Shaf Keshavjee, MD
}

From the Toronto Lung Transplant Program, University of Toronto, Toronto, Ontario, Canada.

Supported by a Canadian Institutes of Health Regenerative Medicine Research Training Award (Frank D'Ovidio).

Read at the Eighty-fourth Annual Meeting of The American Association for Thoracic Surgery, Toronto, Ontario, Canada, April 25-28, 2004.

Received for publication April 23, 2004; revisions received Oct 15, 2004; accepted for publication Oct 21, 2004.

Address for reprints: S. Keshavjee, MD, Director, Toronto Lung Transplant Program, Toronto General Hospital, 200 Elizabeth St, EN10-224, Toronto, Ontario, Canada M5G 2C4 (E-mail: S.Keshavjee@utoronto.ca).

J Thorac Cardiovasc Surg 2005;129: $1144-52$

$0022-5223 / \$ 30.00$

Copyright (๑) 2005 by The American Association for Thoracic Surgery

doi:10.1016/j.jtcvs.2004.10.035
Background: Aspiration of gastroesophageal refluxate may contribute to lung transplant bronchiolitis obliterans syndrome (BOS). We investigated bile acids in bronchoalveolar lavage fluid (BALF) and studied its role in BOS.

Materials and Methods: Surveillance pulmonary function tests and BALF were evaluated in 120 lung recipients. BOS-(0p-3) was diagnosed after 6 months' survival. BOS was defined as "early" if diagnosed within 12 months after a transplant. BALF was assayed for differential cell count, bile acids, and interleukins 8 and 15. Bile acids were considered elevated if greater than normal serum levels $(\geq 8 \mu \mathrm{mol} / \mathrm{L})$.

Results: Elevated BALF bile acids were measured in 20 (17\%) of 120 patients. BOS was diagnosed in 36 (34\%) of 107 patients and judged "early" in $21(57 \%)$ of 36. Median BALF bile acid values were $1.6 \mu \mathrm{mol} / \mathrm{L}$ (range, 0-32 $\mu \mathrm{mol} / \mathrm{L}$ ) in BOS patients and $0.3 \mu \mathrm{mol} / \mathrm{L}$ (range, $0-16 \mu \mathrm{mol} / \mathrm{L}$ ) in non-BOS patients $(P=.002) ; 2.6$ $\mu \mathrm{mol} / \mathrm{L}$ (range, $0-32 \mu \mathrm{mol} / \mathrm{L}$ ) in early BOS patients and $0.8 \mu \mathrm{mol} / \mathrm{L}$ (range, 0-4.6 $\mu \mathrm{mol} / \mathrm{L})$ in late BOS patients, $(P=.02)$. Bile acids correlated with BALF IL-8 and alveolar neutrophilia $(\mathrm{r}=0.3, P=.0004$, and $\mathrm{r}=0.3, P=.004$, respectively), but not with IL-15. Freedom from BOS was significantly shortened in patients with elevated BALF bile acids (Cox-Mantel test, $P=.0001$ ).

Conclusions: Aspiration of duodenogastroesophageal refluxate is prevalent after lung transplantation and is associated with the development of BOS. Elevated BALF bile acids may promote early BOS development via an inflammatory process, possibly mediated by IL-8 and alveolar neutrophilia.

$\mathrm{L}$ ung transplantation is effective therapy for patients with a variety of endstage lung diseases, although the development of posttransplant bronchiolitis obliterans is the major limitation to the long-term success of lung transplantation. Bronchiolitis obliterans (ie, a process of obliteration of the small airways and its clinical correlate) and bronchiolitis obliterans syndrome (BOS; identified as a persistent drop in the forced expiratory volume in 1 second after transplantation) are progressive, respond poorly to augmented immunosuppression, and account for more than $30 \%$ of all deaths occurring after the third postoperative year. ${ }^{1,2}$ Alloimmune-mediated injury directed against endothelial and epithelial structures has been thought to be the underlying cause of bronchiolitis obliterans; however nonalloimmunologic inflammation, including viral infections or ischemic injury, appear also to play a role. ${ }^{3}$ Among the possible inflammatory conditions, aspiration secondary to gastroesophageal reflux (GER) has been suggested to be a potential contributor to lung allograft dysfunction and, in particular, to the development of bronchiolitis obliterans. ${ }^{4,5}$ 
GER disease may be promoted after lung transplantation due to the potential for iatrogenic vagal nerve injury during the course of lung transplantation and to the use of immunosuppressive drugs such as calcineurin inhibitors (cyclosporine and tacrolimus) that cause prolongation of gastric emptying. ${ }^{4-6}$

The association between lung disease and GER disease has been recognized for a long time. ${ }^{7,8}$ In fact GER has been documented to be highly prevalent in patients with a variety of lung diseases: in particular, patients with asthma, cystic fibrosis, and idiopathic pulmonary fibrosis. ${ }^{9,10}$ Furthermore, GER disease has been also associated with the development of bronchiolitis obliterans with organizing pneumonia. ${ }^{11}$ Several methods to document the relationship between lung disease and GER disease have been studied, including scintigraphic monitoring, 24-hour esophageal $\mathrm{pH}$ testing, and assays for pepsin in saliva and sputum. ${ }^{12,13}$ A possible role of duodeno-GER has also been investigated postmortem in sudden infant death syndrome by testing for bile acids within the bronchoalveolar district. ${ }^{14}$ The potential for a role for duodeno-GER is significant in the context of lung transplantation, which is characterized by a high incidence of reduced foregut peristalsis. ${ }^{4}$ We therefore sought to investigate the role of aspiration in this setting by assaying bile acids in bronchoalveolar lavage fluid (BALF) collected during surveillance bronchoscopies and by studying the role of bile acids in the development of BOS. We also studied the relationship between the presence of bile acids within the bronchoalveolar district and other markers that have been associated with the development of BOS. Specifically, we studied the relationship between bile acids and alveolar neutrophilia and interleukin 8 (IL-8) within the BALF. ${ }^{15,16}$ We also studied the interaction with interleukin 15 (IL-15) in an attempt to examine for a possible stimulatory effect of the innate and adaptive immune systems secondary to the presence of bile acids within the bronchoalveolar district. ${ }^{17}$

\section{Material and Methods}

Starting from January 2003, clinical information and excess biological samples (BALF and serum samples) from routine follow-up testing have been collected in 120 lung transplant recipients. BALF was collected from the right middle lobe in bilateral lung transplants and from the right middle lobe or the lingula for single transplants, while transbronchial biopsies (TBBx) were taken from the right upper and right lower lobes in bilateral lung transplants and from the upper and lower lobes in the single lung transplants.

Bronchoscopies with BALF collection and TBBx are routinely performed in the Toronto Lung Transplant Program at 2 and 6 weeks after transplantation, every 3 months for the first year, every 6 months for the second year, and thereafter, as clinically indicated. Thus, data were collected prospectively from consecutive lung transplant patients, although data were also collected from patients who underwent bronchoscopies for diagnostic purposes.

Data were collected regarding BOS development by routine pulmonary function tests. BOS status was determined according to the International Society of Heart and Lung Transplantation grading criteria ${ }^{3}$ : BOS- 0 , absence of the syndrome; BOS-0p-3, presence of 1 of the 4 grades of the syndrome.

For the purpose of this study, patients were required to have been out from transplant at least 6 months before the calculation of the BOS grade and were classified according to the presence or absence of the syndrome; furthermore, the interval of time from transplant to development of BOS was calculated and quantified in months. The time interval from transplant to BOS development was defined as "early" or "late," according to whether BOS-(0p-3) occurred within 12 months of the transplant or not.

The BALF was sent for differential cell count with relative percentage calculation and for microbiology testing. Microbiologic information on the presence or absence of positive bacterial, fungal, or viral cultures was also collected.

The TBBx were assessed for acute rejection and graded according to the Lung Rejection Study Group revised criteria. ${ }^{18}$ The samples were evaluated for presence or absence and grade of rejection and for the presence or absence of signs of inflammation other than acute rejection.

Aliquots of the BALF, taken from the right middle lobe from bilateral and right single lung transplants and from the lingula for left single transplants, were also collected and snap frozen at $-80^{\circ} \mathrm{C}$. The samples were thawed and protease inhibitors (Complete Mini tabs; Boehringer-Mannheim, Mannheim, Germany) were added; the samples were then clarified by centrifugation at $5,000 \mathrm{~g}$ for 10 minutes. The resulting supernatant was assayed for bile acids. The samples were also tested for interleukins 8 (IL-8) and 15 (IL-15) by ELISA with the use of the antibody paired kits (CytoSet kit; BioSource International, Inc, Camarillo, Calif).

To examine the correlation between BALF and serum bile acids, we tested their levels in serum samples $(n=30$ patients $)$ collected within 24 hours of the BALF samples.

The protocol was approved by the Research Ethics Board of the Toronto General Hospital, University Health Network, and informed consent was obtained from each patient for the use of excess BALF and serum, in adherence to the principles set forth in the Helsinki declaration.

\section{Bile Acid Assay}

The bile acid determination in BALF and serum was performed with a spectrophotometric enzymatic assay. The assay was conducted according to the kit instructions (Sigma Diagnostics, Inc, St Louis, Mo). BALF bile acids levels were considered high when they were greater than normal serum levels, ( $\geq 8 \mu \mathrm{mol} / \mathrm{L}$ ), low if they were between 0 to 8 , and absent when they were equal to 0 . Inasmuch as there is no accepted "normal" level of bile acid in BALF, we arbitrarily chose $8 \mu \mathrm{mol} / \mathrm{L}$ (serum upper limit of the normal level range) as a conservative "upper limit of normal."

\section{Statistics}

Statistical analysis was performed with the use of StatView 5 software (StatView, SAS Institute Inc, Cary, NC). Nonparametric statistical analysis of correlation was performed by the Spearman rank correlation test. The Kruskal-Wallis 


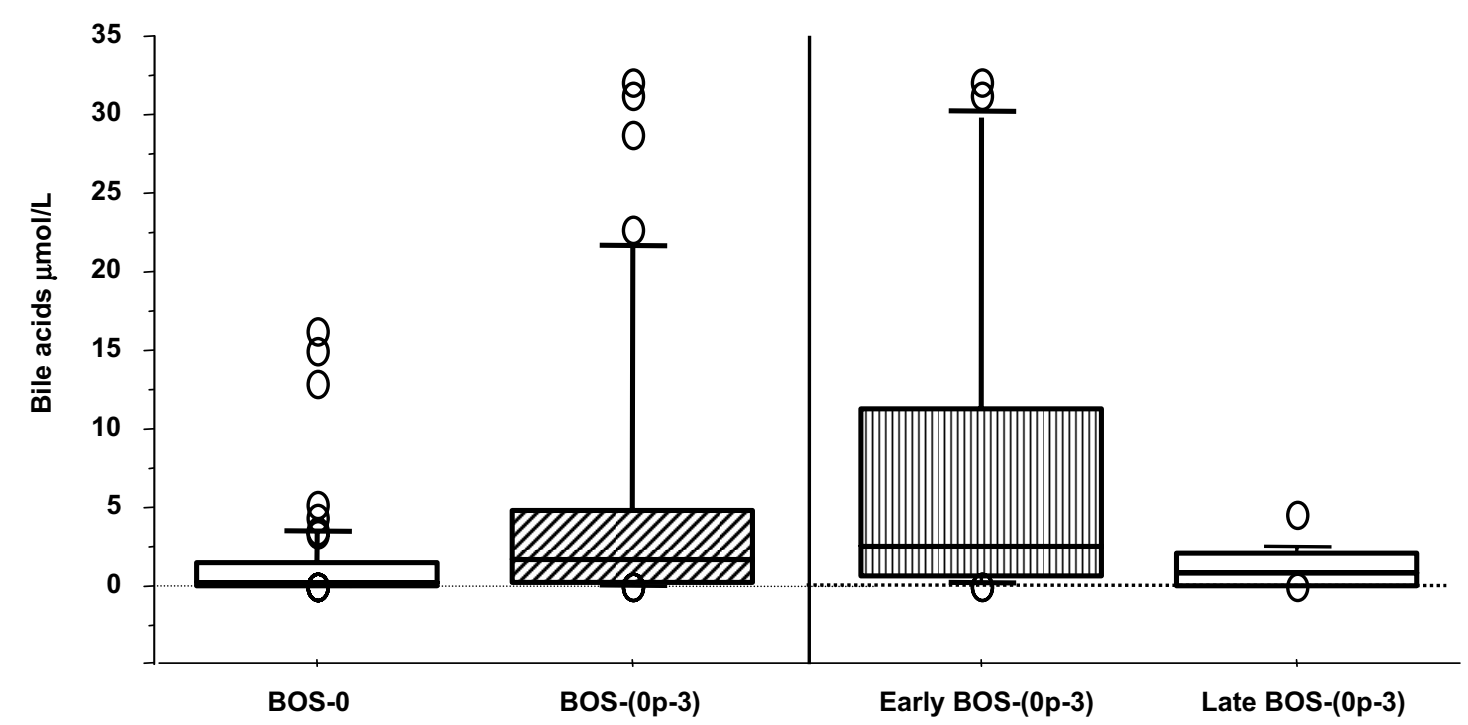

Figure 1. The BALF total bile acids quantified in patients grouped for presence or absence of BOS. The median bile acid level was $0.3 \mu \mathrm{mol} / \mathrm{L}$ (range, $0-16 \mu \mathrm{mol} / \mathrm{L}$ ) for the BOS-0 group and $1.6 \mu \mathrm{mol} / \mathrm{L}$ (range, 0-32 $\mu \mathrm{mol} / \mathrm{L}$ ) in the BOS-(0p-3) group $(P=.002)$. In a stratification of the BOS-(0p-3) group with regard to early ( $\leq 12$ months) versus late (>12 months) BOS development, total bile acids were $2.6 \mu \mathrm{mol} / \mathrm{L}$ (range, 0.06-32 $\mu \mathrm{mol} / \mathrm{L}$ ) and $0.8 \mu \mathrm{mol} / \mathrm{L}$ (range, 0-4.6 $\mu \mathrm{mol} / \mathrm{L})$, respectively $(P=.02)$. The box plots indicate the 25 th, 50 th and 75 th percentile and the bars, the 10th and 90th percentile.

rank test followed by the Mann-Whitney test was used for comparison of continuous variables between groups. Categorical data were analyzed by the $\chi^{2}$ test or the Fisher exact test. Nonparametric actuarial curves, along with the Breslow-Gehan-Wilcoxon test and the Cox-Mantel log-rank test, were used to test the differences in patients grouped with respect to BALF bile acids levels with regard to freedom from BOS-(0p-3) development and with regard to post-lung transplant survival.

\section{Results}

To date, 107 of 120 patients have been assessed with regard to their BOS status, and BOS-(0p-3) was diagnosed in 36 (34\%) of 107 patients: 5 patients with BOS-0p; 14 with BOS-1; 8 with BOS-2; and 9 with BOS-3. The median follow-up times were 14 months (range, 6-46 months) for the patients free of BOS and 24 months (range, 6-125 months) for those with a diagnosis of BOS-(0p-3). Among the 36 patients with a diagnosis of BOS-(0p-3), the syndrome had developed within 12 months of the transplant in 21 patients and was judged "early onset"; in 15 it had developed "late" (after 12 months). The median follow-up time was 18 months (range, 6-40 months) and 36 months (range, 20-125 months) in the early and late groups, respectively, while the median interval of time from transplant to BOS diagnosis was 6.5 months (range, 2-12 months) and 28 months (range, 13-108 months), respectively.
From the 107 patients who were at least 6 months out from transplant and who underwent bronchoscopy, 250 BALF samples were collected. For each parameter studied, namely total bile acids, IL-8 and IL-15, and the differential cell count, the average of the result obtained from the BALF samples of each patient was used as a single value for that patient for the purpose of statistical analysis.

Patients with BOS-(0p-3) status had significantly higher levels of IL-8 (median, $121 \mathrm{pg} / \mathrm{mL}$; range, $0.1-358 \mathrm{pg} / \mathrm{mL}$ ) and IL-15 (median, $26.2 \mathrm{pg} / \mathrm{mL}$; range, 0-109 pg/mL), compared with patients with a BOS-0 status (IL-8: median, 64.5 $\mathrm{pg} / \mathrm{mL}$; range, 0-381; IL-15: median, $15.6 \mathrm{pg} / \mathrm{mL}$; range, $0-615 \mathrm{pg} / \mathrm{mL}), P=.0006$ and $P=.04$, respectively. Comparison between patients with early BOS-(0p-3) and those with late development showed no significant difference for IL-8. In contrast, there were significantly higher levels of IL-15 (median, $39 \mathrm{pg} / \mathrm{mL}$; range, $0-109 \mathrm{pg} / \mathrm{mL}$ ) in the late development group versus the early group (median, 19 $\mathrm{pg} / \mathrm{mL}$; range, $0-101 \mathrm{pg} / \mathrm{mL}), P=.04$.

No correlation between the time points of the BALF collection and those of the IL-8 and IL-15 was noted in all samples $(r=0.2$ and $r=0.1$, respectively; Spearman rank correlation test), although a significant correlation was noted for IL-15 in samples from BOS patients $(\mathrm{r}=0.4, P=$ $.0008)$.

No difference was noted for the BALF differential cell count percentage of neutrophils or lymphocytes when com- 


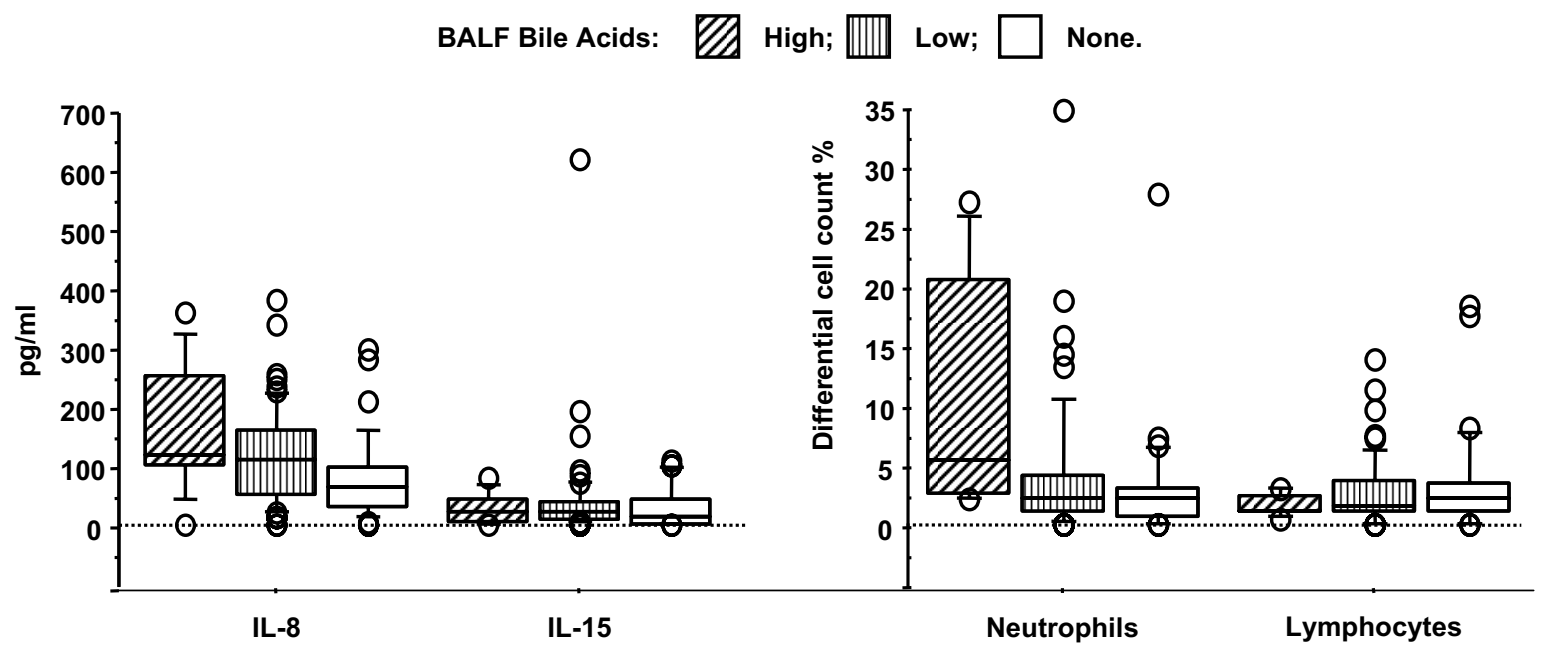

Figure 2. Bile acid within the BALF showed a significant association with IL-8 levels, although no difference was noted for IL-15. The median IL-8 was $118 \mathrm{pg} / \mathrm{mL}$ (range, 1.6-358 pg/mL) for patients with high bile acids, $107 \mathrm{pg} / \mathrm{mL}$ (range, 0-381 pg/mL) for patients with low BALF bile acids, and $61 \mathrm{pg} / \mathrm{mL}$ (range, 0-296 pg/mL) for patients with no bile acids $(P=.01$, Kruskal-Wallis; Mann-Whitney test between no bile versus low bile groups and versus the high bile group showed a $P=.02$ and $P=.01$ respectively). The median IL-15 was $19 \mathrm{pg} / \mathrm{mL}$ (range, 1-80 pg/mL) for high bile acids group, $21 \mathrm{pg} / \mathrm{mL}$ (range, 0-615 pg/mL) for the low bile acids group, and $15 \mathrm{pg} / \mathrm{mL}$ (range, 0-109 pg/mL) for the no bile acid group ( $P=.4$, Kruskal-Wallis test). Bile acid in the BALF showed a significant association with the relative neutrophil cell count but no relationship to the lymphocyte count. When grouped according to bile acid levels, the median neutrophils were $2 \%$ (range, $0 \%-27 \%$ ) in the patients with no bile acids in the BALF; $2 \%$ (range, $0 \%-34 \%$ ) in the group with low BALF bile acids; and $5 \%$ (range, $2 \%-27 \%$ ) in the group with high BALF bile acid ( $P$ $=.015$, Kruskal-Wallis test; Mann-Whitney test for comparison between the high bile acids group versus the low and no bile acids groups showed a $\boldsymbol{P}=.007$ and $\boldsymbol{P}=.008$, respectively). The median lymphocyte count was $2 \%$ (range, $0 \%-18 \%$ ) for the no bile acid group, $1.5 \%$ (range, 0\%-13\%) for the low BALF bile acid group, and $1.1 \%$ (range, $0 \%-3 \%)$ in the high bile acid group $(P=.7$, Kruskal-Wallis). The boxes indicate the 25th, 50th, and 75th percentile and the bars, the 10th and 90th percentile.

paring either BOS-0 to BOS-(0p-3) patients or early versus late BOS-(0p-3) development patients.

The total bile acids quantified in BALF and in serum samples in 30 patients with concurrent collection of the samples showed no correlation $(r=0.067$; Spearman rank correlation test). Therefore, the presence of bile acids within the bronchoalveolar district is not likely due to serum but rather from retrograde aspiration of duodeno-GER.

The average levels of bile acids quantified within the BALF were found to be high $(\geq 8 \mu \mathrm{mol} / \mathrm{L})$ in $10 / 107(9.3 \%)$ patients, low (greater than 0 and less than 8 ) in $61(57 \%)$ of 107 patients. In $36(34 \%)$ of 107 patients, no bile was detected in the BALF.

No correlation between the time points of the BAL collection and the bile acids levels was noted $(r=0.04$; Spearman rank correlation test).

Patients with BOS-(0p-3) had significantly higher bile acids within their BALF than did patients with BOS-0 status (Figure 1). Moreover, as shown in Figure 1, patients in whom BOS-(0p-3) developed early had significantly greater bile acid quantities within the BALF than did the patients in whom BOS developed late. Of the patients with high BALF bile acids, 7 (70\%) of 10 were BOS-(0p-3), and all had the syndrome within the first 12 months from transplant.

A significantly greater quantity of IL- 8 was measured in patients with high levels of bile acids within the BALF, compared with patients with low or absent bile acids. In contrast, no difference was noted with regard to the quantities of IL-15 between the groups, as shown in Figure 2. Patients with high levels of bile acids also had a greater number of alveolar neutrophils than did patients with low bile acids. No difference was noted in the number of alveolar lymphocytes among the groups, as shown in Figure 2.

Analysis of the relationship between bile acids, alveolar neutrophils, IL-8, and IL-15 revealed a moderate correlation between bile acids and alveolar neutrophilia $(\mathrm{r}=0.3 ; P=$ $.005)$ and IL-8 $(\mathrm{r}=0.35 ; P=.0004)$. The correlation for IL-8 also exists in patients with early development of the syndrome $(\mathrm{r}=0.5 ; P=.03)$. No correlation was observed between bile acids and for IL-15 and lymphocyte cell count. 


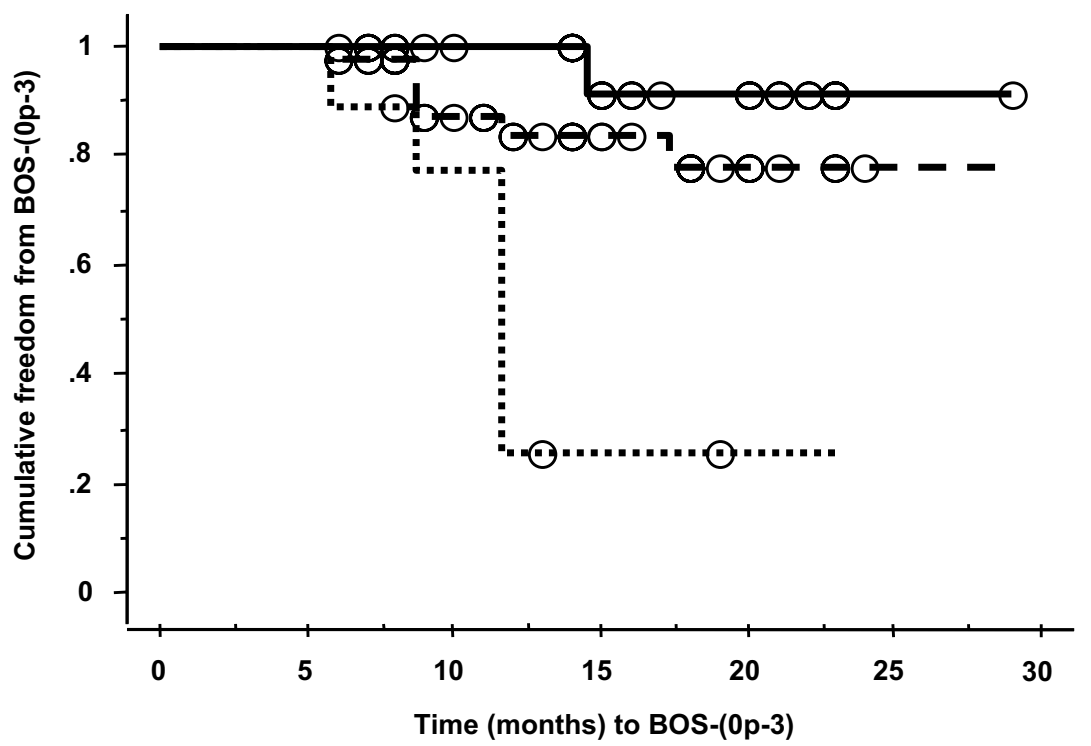

Figure 3. Interval from transplant to development of BOS-(0p-3). The actuarial curves show a significantly reduced interval of time from transplant to BOS-(Op-3) development in patients with high BALF bile acids levels. Breslow-Gehan-Wilcoxon test and CoxMantel log-rank test showed $\boldsymbol{P}=.007$ and $\boldsymbol{P}$ $=.002$, respectively, for the comparison between the group with low bile levels and the group with high bile levels; $P=.0001$ for both tests for the comparison between the group with no detection of bile acids and the group with the high levels of bile acids within the BALF; and $\boldsymbol{P}=.1$ for the comparison between the group with no BALF bile acids and the group with low BALF bile acid levels.
Of the total cohort of patients, 93 of 107 were consecutive patients undergoing lung transplants who survived more than 6 months. In 85 patients, the BALF was collected prospectively before the diagnosis of BOS-(0p-3). BOS(0p-3) was subsequently diagnosed in 15 (18\%) of 85 during the course of this study: 1 patient with BOS-0p; 5 with BOS-1; 4 with BOS-2; and 5 with BOS-3. The median follow-up time was 14 months (range, 6-29 months) for the patients free of BOS and 14 months (range, 6-22 months) for those with a diagnosis of BOS-(0p-3). In the latter group, the median interval of time from transplant to BOS(0p-3) diagnosis was 9 months (range, 3-16 months).

The time to BOS-(0p-3) development from the transplant was quantified in months and used to determine the actuarial freedom from BOS development; the 85 patients with prospective data collection were grouped according to levels of bile acids (Figure 3 ). The curves were generated from the data pertaining to the cohort of 85 patients in which the BALF fluid samples were collected prospectively from the time of transplant. In the BOS-(0p-3) patients, only bile acid quantification results from BALF samples collected before BOS development were used for the analysis. Of these 85 patients, 33 did not have any bile acid detected in the BALF, 43 patients had a total bile acid concentration between 0 to $8 \mu \mathrm{mol} / \mathrm{L}$, and 9 patients had bile acid readings greater than or equal to $8 \mu \mathrm{mol} / \mathrm{L}$.

From the 120 patients, 272 BALF samples were studied. Of these, 261 had microbiology reports. Of all the concomitant transbronchial biopsies performed, 184 of 272 were adequate for the determination of the presence or absence of acute rejection, and 203 were adequate for determination of signs of inflammation. In BALF samples grouped with regard to the presence or absence of signs of inflammation in the concomitant $\mathrm{TBBx}$, samples with inflammation showed a median $1.1 \mu \mathrm{mol} / \mathrm{L}$ bile acids (range, $0-54 \mu \mathrm{mol} /$ L), and samples negative for inflammation showed a median $0.2 \mu \mathrm{mol} / \mathrm{L}$ of bile acids (range, $0-71 \mu \mathrm{mol} / \mathrm{L}$ ), $P=.007$. When the samples were grouped for the presence or absence of acute rejection diagnosed from concomitant TBBx, no difference was noted.

BALF samples grouped according to microbiology culture findings showed a significantly greater quantity of bile acids in BALF samples positive for bacterial growth (median, $0.7 \mu \mathrm{mol} / \mathrm{L}$; range, $0-71 \mu \mathrm{mol} / \mathrm{L}$ ) than in the samples negative for bacteria (median, $0.3 \mu \mathrm{mol} / \mathrm{L}$; range, 0-52 $\mu \mathrm{mol} / \mathrm{L} ; P=.02$ ). Similarly, bile acid quantities were significantly greater in samples positive for fungal growth (median, $0.75 \mu \mathrm{mol} / \mathrm{L}$; range, $0-57 \mu \mathrm{mol} / \mathrm{L}$ ) than in the samples negative for fungus (median, $0.36 \mu \mathrm{mol} / \mathrm{L}$; range, $0-71 \mu \mathrm{mol} / \mathrm{L} ; P=.048)$. No significant association was seen in relation to the presence of cytomegalovirus in the BALF.

\section{Discussion}

Over the past decade, improvements in surgical techniques, lung preservation, immunosuppression, and management of ischemia-reperfusion injury and infections have contributed to an increase in the 1-year patient survival after lung transplantation to $70 \%$ to $80 \% .{ }^{19}$ However, long-term survival continues to be threatened by the development of 
bronchiolitis obliterans, or chronic lung dysfunction. The clinical correlate, BOS was identified as a persistent drop in the forced expiratory volume in 1 second after transplantation, which affects $50 \%$ to $60 \%$ of patients at 5 years after transplantation. ${ }^{2}$

Five-year survival after the onset of bronchiolitis obliterans is only $30 \%$ to $40 \%$, and survival at 5 years after transplantation is $20 \%$ to $40 \%$ lower in patients with bronchiolitis obliterans. ${ }^{20}$

Acute and chronic onset of BOS has been described, suggesting that BOS reflects at least 2 modes of onset with different natural histories. ${ }^{21}$ Interestingly, 2 forms of bronchiolitis obliterans have been described in heart-lung transplant recipients: a relatively acellular concentric fibrosing process limited to the terminal bronchioles; and a focal and cellular process extending into the distal alveolar spaces, and associated with aspirated material and foreign bodytype giant cells. ${ }^{22}$ The latter pathologic finding is indeed supportive of a possible role for GER in the development of bronchiolitis obliterans. ${ }^{4,5}$

A possible role of retrograde aspiration after GER in the early development type of BOS has been implicitly documented in the recent work from Cantu and colleagues, ${ }^{23}$ in which they show that, in lung transplant patients with known GER disease, early fundoplication led to significant improvements in freedom from the development of BOS.

GER disease has been also associated with the development of bronchiolitis obliterans-associated pneumonia or diffuse bronchiolitis in the non-lung transplant population. $^{11,24}$

In lung transplant patients, pulmonary defense mechanisms, including the cough reflex and mucociliary clearance of foreign bodies, are markedly impaired. Mucociliary clearance has been measured to be less than $15 \%$ of normal in transplanted lungs. ${ }^{4}$ It is conceivable that a prolonged contact time of aspirated gastric contents may therefore lead to substantially greater lung parenchymal injury. Moreover, while GER may cause direct lung injury, it is also possible that it may play a role in augmenting the alloimmune response by creating an upregulated inflammatory milieu locally. ${ }^{5}$

Bile aspiration secondary to duodeno-GER has been associated with severe pulmonary injury ${ }^{25}$ and the cytotoxicity may result in the disruption of the cellular membrane or in the alteration of cellular cationic permeability depending on the bile acid concentration, as Oleberg and colleagues ${ }^{26}$ demonstrated in vitro using type II pneumocytes.

The potential for a significant role for aspiration secondary to duodeno-GER is prominent in the context of lung transplantation, which is characterized by a high incidence of reduced foregut peristalsis. ${ }^{4}$ Moreover, chronic silent acid aspiration per se may not be as injurious as aspiration of other components of the refluxate. ${ }^{27}$ We have attempted
Table 1. Distribution frequency of patients grouped according to bile acids levels and the presence or absence of BOS-(Op-3), and according to early versus late development of BOS-(0p-3) $\left(P=.009\right.$ and $P=.02$, respectively; $\chi^{2}$ test)

\begin{tabular}{lcccrrr}
\hline & \multicolumn{5}{c}{ BOS and BALF bile acid association } \\
\cline { 2 - 7 } & BOS-0 & BOS-(Op-3) & Total & Early & Late & Total \\
\hline No bile acids & 29 & 7 & 36 & 2 & 5 & 7 \\
Low bile acids & 39 & 22 & 61 & 12 & 10 & 22 \\
High bile acids & 3 & 7 & 10 & 7 & 0 & 7 \\
Total & 71 & 36 & 107 & 21 & 15 & 36 \\
\hline
\end{tabular}

to objectively investigate aspiration by assessing for presence of bile acids in BALF collected during surveillance bronchoscopies and have studied its potential contribution to the development of BOS. Bile acids were investigated as markers of duodeno-GER, rather than as a causative agent of the refluxate. Other components of the duodenal and gastric juices, such as pepsin and trypsin, could be implicated, as long as one considers that the bronchoalveolar district has a $\mathrm{pH}$ that favors the activity of the duodenopancreatic agents, rather than the gastric ones.

One would intuitively expect that no bile acids would be detectable in the lung under normal conditions. However, the levels of bile acids in vivo within the bronchoalveolar district have not been previously reported; thus, no levels of normality have been determined. We therefore chose to use the upper limit of normal for serum bile acids $(\leq 8 \mu \mathrm{mol} / \mathrm{L})$ as "normal" for the purpose of this study.

On examining BALF and serum samples collected within 24 hours of the bronchoscopy, we noted no correlation for quantities of total bile acids, suggesting that readings within fluid collected from the bronchoalveolar district is secondary to retrograde aspiration, rather than to serum bile acid. We have also noted a relationship between BALF bile acids and abnormal $\mathrm{pH}$ findings in lung transplant patients. ${ }^{28}$

The data collected in our study clearly defined an early and late BOS development group of patients with specific biological characteristics, thus supporting the existence of 2 types of onset of BOS. There is a definite association between reduced time to BOS development and levels of bile acids detected within the bronchoalveolar district. The early BOS patients in our study had a greater content of bile acids within their BALF samples, compared to the late BOS group. Of the patients with high BALF bile acids levels, $70 \%$ had BOS-(0p-3), and in all the syndrome had developed within the first 12 months from surgery. Table 1 shows the distribution of the patients according to the level of bile acids and BOS grade, and according to the time to development of BOS. 
The biological mechanism by which the bile acids contribute to lung graft dysfunction appears to be mediated by IL-8 and alveolar neutrophilia. Interestingly, both Il-8 and alveolar neutrophilia have been previously described as putative clinical markers of BOS development. ${ }^{15,16}$ Moreover, in patients with early BOS development, the relationship between bile acids and IL- 8 seems to be particularly strong.

Interestingly, the late BOS group was found to have a significantly greater content of IL-15. IL-15 is involved in both innate and adaptive immunity with specific activity toward lymphocyte proliferation. IL-15 also functions as an antigen-independent activator of CD8 memory $\mathrm{T}$ cells, which implies a possible different immunologic mechanism involved in late versus early BOS development. ${ }^{17}$

The mechanism by which bile acids might contribute to lung graft dysfunction was not the primary focus of this study, although some hypotheses could be generated by the significant association within the BALF between bile acid and bacterial and fungal infection, and between the presence of bile acids and the TBBx findings related to the diagnosis of acute interstitial inflammation.

Bile acids act as barrier breakers within other organs, such as the gastric mucosa, where they are known to disrupt the surfactant layer that protects the mucosa. ${ }^{29,30}$ In a similar fashion, this may well be happening within the bronchoalveolar district with bile acids possibly functioning as detergents to disrupt the lipid layers of the pulmonary surfactant. Alternatively, bile acids may cause direct injury to type II pneumocytes that are responsible for the production and homeostasis of the pulmonary surfactant and its related proteins. ${ }^{26}$ Thus, by disrupting locoregional innate immunity, bile acids may consequentially promote infections and/or maintain chronic infections. Furthermore, they could be responsible for upregulation of the adaptive immune response, given that the 2 systems, innate and adaptive, are in a continuous balance adjustment. Thus, a loss of effective innate immunity may lead to a more aggressive adaptive immune response. ${ }^{31}$ Further studies are required to validate these mechanistic hypotheses.

\section{Conclusion}

Our findings strongly support a significant role for GER disease in the development of BOS after lung transplantation. We have documented that lung transplant patients with bile in their BALF as a marker of duodeno-GER aspiration have a reduced freedom from BOS. Importantly, patients with high levels of bile acids in the BALF develop BOS earlier. Elevated bile acids in the BALF of these patients are associated with elevated IL-8 levels and increased BALF neutrophilia. BOS is a devastating syndrome that affects lung transplant patients. Clearly, its pathogenesis is multifactorial. The data obtained from this study provide addi- tional objective evidence implicating a contributory role of duodeno-GER in the development of BOS. Bile acids were investigated as markers of retrograde aspiration, although a temporal link, along with a dose-response effect, was observed with the development of BOS. Furthermore, bile acids carry a plausible biological activity toward lung injury ${ }^{25,26,29,30}$; thus, this study provides information that also supports their active role, whether primary or contributive to other agents, toward the development of bronchiolitis obliterans.

We hope that further objective evidence such as this will help us to determine which lung transplant patients might benefit from antireflux therapy to prevent at least 1 component of the ongoing injury to the transplanted lung.

\section{References}

1. Burke CM, Theodore J, Dawkins KD, Yousem KD, Blank N, Billingham ME, et al. Posttransplant obliterative bronchiolitis and other late lung sequelae in human heart-lung transplantation. Chest. 1984;86: 824-9.

2. Boehler A, Kesten S, Weder W, Speich R. Bronchiolitis obliterans after lung transplantation: a review. Chest. 1998;114:1411-26.

3. Estenne M, Maurer JR, Boehler A, et al. Bronchiolitis obliterans syndrome 2001: an update of the diagnostic criteria. J Heart Lung Transplant. 2002;21:297-310.

4. Berkowitz H, Schulman LL, McGregor C, et al. Gastroparesis after lung transplantation-potential role in postoperative respiratory complications. Chest. 1995;108:1062-7.

5. Davis RD, Jr, Lau CL, Eubanks S, Messier RH, Hadjiliadis D, Steele $\mathrm{MP}$, et al. Improved lung allograft function after fundoplication in patients with gastroesophageal reflux disease undergoing lung transplantation. J Thorac Cardiovasc Surg. 2003;125(3 Pt 1):533-42.

6. Young LR, Hadjiliadis D, Davis RD, Palmer SM. Lung transplantation exacerbates gastroesophageal reflux disease. Chest. 2003 Nov;124(5): 1689-93.

7. Pearson JEG, Wilson RSE. Diffuse pulmonary fibrosis and hiatus hernia. Thorax. 1971;26:300-30.

8. Belsey R. The pulmonary complications of dysphagia. Thorax. 1948; 4:44-56.

9. Feigelson J, Girault F, Pecau Y. Gastro-oesophageal reflux and esophagitis in cystic fibrosis. Acta Paediatr Scand. 1987;76:989-90.

10. Tobin RW, Pope CE $2^{\text {nd }}$, Pellegrini CA, Emond MJ, Sillery J, Raghu G. Increased prevalence of gastroesophageal reflux in patients with idiopathic pulmonary fibrosis. Am J Respir Crit Car Med. 1998;158: 1804-8.

11. Sadoun D, Valeyre D, Cargill J, Volter F, Amouroux J, Battesti JP. Bronchiolitis obliterans with cryptogenetic-like organizing pneumonia. Demonstration of gastro-esophageal reflux in 5 cases. Presse Medicale. 1988;17:2383-5.

12. Ghaed N, Stein M. Assessment of a technique for scintigraphic monitoring of pulmonary aspiration of gastric contents in asthmatics with gastroesophageal reflux. Ann Allergy. 1979;42:306.

13. Potluri S, Friedenberg F, Parkman HP, et al. Comparison of a salivary/ sputum pepsin assay with 24-hour esophageal $\mathrm{pH}$ monitoring for detection of gastric reflux into the proximal esophagus, oropharynx and lung. Dig Dis Sci. 2003;48:1813-7.

14. Hills BA, Chen Y, Masters IB, Hills Y. Raised bile acid concentration in SIDS lungs at necroscopy. Arch Dis Childhood. 1997;77:120-3.

15. Devouassoux G, Drouet C, Pin I, et al. Alveolar neutrophilia is a predictor for the bronchiolitis obliterans syndrome and increases with degree of severity. Transplant Immunology. 2002;10:303-10.

16. DiGiovine B, Lynch III JP, Martinez FJ, et al. Bronchoalveolar lavage neutrophilia is associated with obliterative bronchiolitis after lung transplantation. Role of Il-8. J Immunol. 1996;157:4194-202. 
17. Weng NP, Liu K, Catalfamo M, Li Y, Henkart PA. IL-15 is a growth factor and an activator of CD8 memory T cels. Ann NY Acad Sci. 2002;975:46-56

18. Yousem SA, Berry GJ, Cagle PT, Chamberlain D, Husain AN, Hruban $\mathrm{RH}$, et al. Revision of the 1990 working formulation for the classification of pulmonary allograft rejection: Lung Rejection Study Group. J Heart Lung Transplant. 1996;15(1 Pt 1):1-15.

19. Hosenpud JD, Bennet LE, Keck BM, Boucek MM, Novick RJ. The Registry of the International Society for Heart and Lung Transplantation: eighteenth official report-2001. J Heart Lung Transplant. 2001; 20:805-15.

20. Valentine VG, Robbins RC, Berry GJ, Patel HR, Reichenspurner H, Reitz BA, et al. Actuarial survival of heart-lung and bilateral sequential lung transplant recipients with obliterative bronchiolitis. J Heart Lung Transplant. 1996;15:371-83.

21. Sharples LD, Jackson CH, McNeil K, Wallwork J. Acute and chronic onset of bronchiolitis obliterans syndrome (BOS): are they different entities? J Heart Lung Transplant. 2001;20:197.

22. Abernathy EC, Hruban RH, Baumgartner WA, et al. The two forms of bronchiolitis obliterans in heart-lung transplant recipients. Hum Pathol. 1991;22:1102-10.

23. Cantu E III, Appel JZ III, Hartwig MG, Woreta H, Green C, Messier $\mathrm{R}$, et al. Early fundoplication prevents chronic allograft dysfunction in patients with gastroesophageal reflux disease. Ann Thorac Surg. 2004; 78:1142-51; discussion 1142-51.

24. Matsuse T, Oka T, Kida K, Fukuchi Y. Importance of diffuse aspiration bronchiolitis caused by chronic occult aspiration in the elderly. Chest. 1996;110:1289-93.

25. Porembka DT, Kier A, Sehlhorst S, Boyce S, Orlowski JP, Davis K Jr. The pathophysiologic changes following bile aspiration in a porcine lung model. Chest. 1993;104:919-24.

26. Oelberg DG, Downey SA, Flynn MM. Bile salt induced intracellular $\mathrm{Ca}++$ accumulation in type II pneumocytes. Lung. 1990;168:297-308.

27. Effros RM, Hogan G, Hoagland KW, Olson L, Lin W. Protection of the lungs from acid during aspiration. Am J Med. 2001;111(8A):56S59 S.

28. D'Ovidio F, Mura M, Waddell TK, et al. Bile acids in bronchoalveolar lavage after lung transplantation as a marker of pulmonary aspiration associated with alveolar neutrophilia. J Heart Lung Transplant. 2004; 23(2 Suppl 1):42S.

29. Hills BA, Butler BD, Lichtenberger LM. Gastric mucosal barrier: hydrophobic lining to the lumen of the stomach. Am J Physiol. 1983; 244(7): G561-8

30. Vaezi MF, Richter JE. Duodenogastroesophageal reflux and methods to monitor nonacidic reflux. Am J Med. 2001;111(8A):160S-8S.

31. Medzhitov R, Janeway C. Innate immunity. N Engl J Med. 2000;343: $338-44$.

\section{Discussion}

Dr Yolonda Colson (Boston, Mass). Very nice. In terms of mechanism, do you think that the bile acids themselves are causing the injury, do you think it's a marker for other things in the reflux that are causing it, or do you think it's actually activating macrophages, neutrophils, whatever, in the lungs to then lead on to the injury?

Dr D'Ovidio. As of now, we can consider it only as a marker, although our working hypothesis is that bile acid per se or other components of the refluxate are interacting within the bronchoalveolar district, in particular with surfactant. We have preliminary data showing a significant association of bile acid and surfactant proteins $\mathrm{A}$ and $\mathrm{D}$ and an association between bile acid and disruption of the phospholipid layers, in particular with a reduced quantity of DPPC and PG and an elevation of sphingomyelin and lysoPC, the primary ones which are tensoactive and the later ones which are markers of inflammatory status. Bile acids are cytotoxic - we know that from the hepatobiliary literature - and are barrier-breakers, therefore may function as the key players.
Dr Raphael Bueno (Boston, Mass). That was a very nice talk. It continues to support the thesis that reflux harms lung transplant patients. I have a few questions. Do the patients who are at high risk for BOS and have a high reflux have a particular disease that you transplant them for, or are they all comers? I mean is it mostly patients with idiopathic pulmonary fibrosis and cystic fibrosis who have reflux?

Dr D'Ovidio. They are all comers, and, in a smaller cohort of patients, we have $\mathrm{pH}$ testing results showing no difference with regard to the incidence of $\mathrm{pH}$-metry abnormalities within the different end-stage lung disease for which they were transplanted. Furthermore, in the same cohort of patients, we did gastric emptying studies, and, in those, there was an average of about $78 \%$ of prolonged gastric emptying for solids, which was equally distributed for restrictive rather than obstructive end-stage lung disease. I think there is a posttransplant high incidence of gastroesophageal reflux disease, and it's in about $50 \%$ of our patients with regard to pH-metry. There is a significant gastric emptying delay factor, which is in over $70 \%$ of the patients. Now, whether all of these are aspirating, probably not, and that's why we tried to document aspiration objectively by using bile acid as a marker.

Dr Bueno. Let me understand this. Your hypothesis is that bile aspiration is a more sensitive test for future BOS than, for example, $\mathrm{pH}$ manometry or symptoms of reflux?

Dr D'Ovidio. It may be more specific, because not all $\mathrm{pH}$ tested patients had elevated bile acids within the BALs. We noted an association between the presence of high bile acid content in BALs and abnormal proximal esophageal $\mathrm{pH}$-metry, but not with distal pH-metry abnormalities within the esophagus.

Dr D. Davis (Durham, NC). I'm going to take you in a different direction, essentially the ones that have zero or have no bile in it. Does that mean that we should be wrapping absolutely everybody? I mean obviously we have had an interest in only those that had pretty significant reflux by $\mathrm{pH}$ testing. Does this mean that we really need to be shooting for zero and that everybody should be wrapped at the time that they get a lung transplant?

Dr D'Ovidio. I'm not sure we should be aiming for zero, because we don't know what the normal values within the BAL samples of a normal patient population should be. Certainly bile acid is a marker. I'm not sure it's the best marker of aspiration from the gastric content. Maybe pepsin or trypsin might work out better and have a greater correlation with even the distal $\mathrm{pH}$ testing, rather than the proximal $\mathrm{pH}$ testing within the esophagus.

I think that maybe not all $\mathrm{pH}$-abnormal patients should be wrapped. Probably a good portion of the patients will react to prokinetic agents and not necessarily need wrapped. But definitely if we consider this a valid marker, we should be quite aggressive in treating whatever we can, because there is, as I've shown, a temporal link with the elevation of bile acid in the BALs and the more aggressive and early development of BOS.

Dr Davis. Well, I guess that's the question. What you're showing has nothing to do with what's normal or abnormal. What you're showing or implicating is that any is bad and that we really should not be looking at normality but we really should be looking at creating almost a superphysiologic prevention.

Dr D'Ovidio. Well, I do not think we're showing that any is detrimental, because within that low group of patients there is a range between 0 and 8 , and I suspect that the true cutoff should be 
more at around 3 or 4 based at least on the raw data I have. I have not tried to determine which would be a better cutoff by statistics.

Dr Davis. I thought your data on the zero, though, was underpowered, but it looked significantly better than even your, quote/ unquote, "low."

Dr D'Ovidio. True, but there are in our group of patients samples with a range of 0 to $1 \mu \mathrm{mol} / \mathrm{L}$ which had no BOS development within the time frame we assessed them.

Dr Sandro Mattioli (Bologna, Italy). Eighty percent of bile acids which reflux in the stomach are glycoconjugated acids, which turn in protonated form in an acid environment. In the protonated form, bile acids have very low solubility and activity. Contrarily, in an acidic or alkaline environment, glycoconjugated bile acids are in ionized, highly soluble, and active form .You were correct when you said bile acids are at the present time mainly a marker of reflux. We don't' know its pathologic mechanisms, if there are any, when the stomach is normally secreting. So my question is were those patients submitted to chronic acid suppression therapy with proton pump inhibitors after transplantation in order to understand if they had an acid, acidic or alkaline stomach.

Dr D'Ovidio. Postoperatively, all of our patients are on proton pump inhibitors or $\mathrm{H}_{2}$-blockers. On the other hand, the bile acids within the bronchoalveolar district would be in a nonacidic environment. Therefore, they could change their presentation, and they may be activated.

\section{Bound volumes available to subscribers}

Bound volumes of The Journal of Thoracic and Cardiovascular Surgery are available to subscribers (only) for the 2005 issues from the Publisher, at a cost of $\$ 134.00$ for domestic, $\$ 165.85$ for Canadian, and $\$ 155.00$ for international subscribers for Vol 129 (January-June) and Vol 130 (July-December). Shipping charges are included. Each bound volume contains a subject and author index.

The binding is durable buckram with the Journal name, volume number, and year stamped in gold on the spine. Payment must accompany all orders. Contact Elsevier Inc., Subscription Customer Service, 6277 Sea Harbor Dr, Orlando, FL 32887, USA; phone $800-654-2452$ or $407-345-4000$.

Subscriptions must be in force to qualify. Bound volumes are not available in place of a regular Journal subscription. 